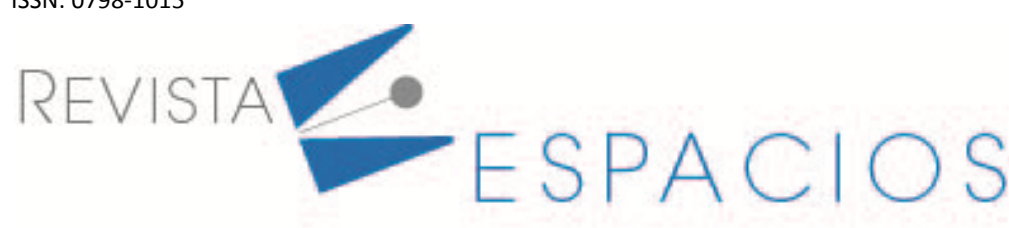

\title{
Apreciación de la enseñanza-aprendizaje online en tiempos de COVID-19 por parte de las familias españolas
}

\section{Appreciation of online teaching-learning in times of COVID-19 by Spanish families}

\author{
CABRERA-MÉNDEZ, Margarita ${ }^{1}$ \\ DÍEZ-SOMAVILLA, Rebeca \\ LÓPEZ-NAVARRETE, Alberto J. ${ }^{3}$
}

\begin{abstract}
Resumen
El estudio analiza la satisfacción de las familias españolas con hijos en educación obligatoria (de 6 a 16 años) tras el paso de docencia presencial a docencia digital a causa del COVID-19. Mediante un muestreo aleatorio simple de 2.150 individuos de toda España: se observan diferencias de satisfacción entre las familias que llevan a sus hijos a un centro público, concertado o privado; también analiza la impresión sobre la metodología del sistema de enseñanza en el nuevo contexto.

Palabras clave: educación online, educación primaria, educación secundaria, COVID-19
\end{abstract}

\begin{abstract}
The study analyses the satisfaction of Spanish families with children in compulsory education (from 6 to 16 years old) after the change from face-to-face to digital teaching due to the COVID-19. Using a simple random sampling of 2,150 individuals from all over Spain: differences in satisfaction are observed among families who take their children to a public, subsidized, or private center; it also analyzes the impression on the methodology of the teaching system in the new context.

key words: online education, primary education, secondary education, COVID-19
\end{abstract}

\section{Introducción}

El estudio examina las percepciones de padres y madres de alumnos en educación obligatoria en España (Educación Primaria y Educación Obligatoria Secundaria, ESO) en relación a la experiencia de haber vivido el paso de una docencia presencial a una docencia digital. La pandemia de la COVID-19 ha supuesto un cambio abrupto e inmediato que ha generado «una enseñanza remota de emergencia» (Hodges et al., (2020).

El objetivo es principal es conocer el grado de satisfacción de las familias con la formación online y su percepción en cuanto a este cambio educativo, en una situación generalizada de teletrabajo y de crisis económica y sanitaria. Como objetivos secundarios se plantea conocer si existe diferencia en el grado de satisfacción de las familias que llevan a sus hijos a un centro público, a uno concertado o a uno privado, así como si existen diferencias entre primaria y secundaria en esta apreciación. También se quiere identificar el nivel de conocimiento de las familias

\footnotetext{
${ }_{1}^{1}$ Profesora titular. Comunicación Audiovisual, Documentación e Historia del Arte . Universitat Politècnica de València. mcabrera@upv.es

2 Profesora ayudante doctor. Comunicación Audiovisual, Documentación e Historia del Arte. Universitat Politècnica de València. rdiez@har.upv.es

3 Técnico superior de investigación. Comunicación Audiovisual, Documentación e Historia del Arte. Universitat Politècnica de València. allona2@upv.es
} 
de la formación en línea de sus hijos y la impresión sobre la metodología del sistema de enseñanza en el nuevo contexto.

El 14 de marzo de 2020, el Gobierno español decreta el estado de alarma y, tal como se recogió en el BOE-A2020-3692, «todos los centros educativos quedan cerrados de manera total a la actividad académica [...] durante el período de suspensión se mantendrán las actividades educativas a través de las modalidades a distancia y en línea, siempre que resulte posible». El cierre de colegios puede ocasionar efectos negativos (Sanz, 2020), pero pueden atenuarse si existe una respuesta coordinada y no se alarga dicha suspensión en el tiempo (Jaume \& Willén, 2019). Este cierre provocó el cambio repentino de una enseñanza-aprendizaje presencial a la modalidad online, y destapó numerosas incertidumbres en un territorio en el que las competencias sobre educación están repartidas entre el Estado y las 17 Comunidades Autónomas. Además de una legislación dispar, durante la primera semana de docencia se detectó que «no había tiempo ni espacio para la aplicación y el diseño de las experiencias de aprendizaje que caracterizan una auténtica educación online» (Zubillaga \& Gortazar, 2020). Se plantearon cuestiones sobre la coordinación de las tareas, canales de envío, dificultades de conexión, brecha de acceso, nuevos sistemas evaluativos, etc. La educación se vio inmersa en una situación de crisis, entendida como problema o mala situación (Coombs, 1978). Una crisis en la que los centros intentan adaptar su enseñanza y esperan las directrices del Ministerio de Educación, mientras las familias se preparan a una transición educativa compleja y online. El informe Cotec (Zubillaga \& Gortazar, 2020) asegura que el cierre de las escuelas producirá un impacto negativo sobre el aprendizaje de todos los alumnos.

Nace así la idea de preguntar a los padres su percepción en el cambio educativo y poder analizar, en futuras investigaciones, la evolución de la formación online implantada en tiempo récord a nivel nacional, como base para posibles mejoras: variación de horarios y tiempo dedicado de los escolares a su formación, la relación entre educación y aprendizaje, o las desigualdades entre instituciones públicas, concertadas y privadas. Además, se quiere llegar a una identificación de la percepción que tienen las familias sobre la implicación y esfuerzo de los docentes en todo este proceso y cómo creen que lo están viviendo los menores. El estudio destapa las percepciones en cuanto a la inmersión súbita de la docencia online y la implicación de los docentes y alumnos. En las conclusiones se discute el cambio exprés hacia la docencia online en la que se ha visto inmersa España, y en qué medida se puede mejorar de cara al futuro según los resultados analizados, teniendo en cuenta que el Ministerio de Educación y las autonomías prevén un modelo escolar mixto para el curso 20-21, online y presencial (Zafra, 2020).

\subsection{Contexto social-educativo COVID-19}

La COVID-19 ha sumido al mundo en una crisis sin precedentes en la historia contemporánea a nivel sanitario, económico, político y social. De entre los diferentes estilos de educación (González-Peiteado \& Pino-Juste, 2016), en España se parte de una enseñanza basada en la docencia tradicional en la que el profesor es el protagonista (Ballesta-Pagan et al., (2011: 356) y el aprendizaje de los alumnos depende de lo que los profesores saben y pueden hacer (Darling-Hammond, 2000). Sin embargo, la situación de pandemia exige una transformación digital súbita, pasando de lo presencial a lo digital en tan solo unos días para ajustar, programar y reprogramar los calendarios escolares.

El 14 de mayo se decreta el estado de alarma y el cierre de centros educativo; el lunes 16 se inicia una nueva fase de enseñanza online y digital en un contexto de escasa formación digital de los docentes (Fernández-Cruz \& Fernández-Díaz, 2016; Cabrera-Méndez et al., 2019), con estudiantes rodeados de equipos electrónicos que han configurado su vida (Feixa, 2006:13), pero sin saber utilizar las tecnologías digitales (Pérez-Escoda et al., 2016).

El informe Cotec 2020 duda de la consecución de los objetivos educativos para el curso 2019-2020, ya que la crisis no deja tiempo ni espacio para la planificación de una auténtica educación online, teniendo en cuenta que 
en España solo el 50\% de los centros están digitalizados (PISA, 2018). El problema se centra sobre tiempo y espacio, pero otros factores han de tenerse en cuenta para realizar una radiografía educativa en el nuevo sistema y poder realizar los ajustes oportunos para el próximo curso.

\subsection{De la educación presencial a la enseñanza online: carencias en la alfabetización digital}

El cierre de los centros ha provocado el paso repentino a una educación a través de dispositivos electrónicos como herramientas para mejorar el acceso a la formación, la comunicación y la interacción, denominado eLearning (Sangrá et al., ( 2012).

La tecnología se ha convertido en un requisito para la educación (Moore \& Kearsley, 2011); ya era parte de la vida cotidiana de los jóvenes (Tapscott, 1999), sobre la que configuran su visión del mundo (Feixa, 2006) y al que Bringué-Sala y Sábada-Chalezquer (2010) calificaron de «multitodo». Jóvenes denominados nativos digitales (Prensky, 2001), término aplicado primero a la generación millenial, y después a la generación Z que han sido objeto de estudio sobre el conocimiento de las competencias digitales que se les atribuía (Ala-Mutka, 2011. Desde un punto de vista socio-cognitivo, la generación $\mathrm{Z}$ se caracteriza por una capacidad de respuesta rápida; deseo de inmediatez e interacción continua; autoconcebidos como expertos en TIC, atribuyendo grandes expectativas a la tecnología donde el aprendizaje suele ser independiente o autodidacta (Fernández \& Fernández, 2016), con un acceso temprano (Feixa, 2006) y mayor precocidad de los niños en estar de modo intensivo frente a las pantallas (Blanco \& Römer, 2011).

Pronto se concluyó que estas características hacia la tecnología no suponen el desarrollo de la competencia digital (Gilster, 1997; Pérez-Escoda et al., 2016). Varios autores hablan de la falacia del nativo digital (CabraTorres \& Marciales-Vivas, 2009; Cobo \& Moravez, 2011; Johnson et al. 2014) por el nivel insuficiente de competencia digital en niños y adolescentes europeos. El niño no adquiere habilidades digitales de forma inherente, sino que precisa de educación al respecto (Cabero-Almenara \& Marín-Díaz, 2014).

\subsection{Características tecnológicas de los centros y del ámbito familiar: la brecha digital}

Tecnológicamente, a priori, España no está mal situada. El 91,4\% de los hogares españoles tiene conexión a Internet, según los datos del Instituto Nacional de Estadística de 2019. Sin embargo, nunca se había vivido un simulacro de teletrabajo y teleformación en familias para el que no se estaba preparado. Al inicio del estado de alarma, los principales operadores de telefonía pedían mesura en el uso de las conexiones para dar prioridad al teletrabajo (McLoughlin, 2020). Según El Estudio de Menores y TIC (2019), el 92,8\% de los menores (10-15 años) utiliza Internet de forma habitual, el ordenador (91,3\%), tienen móvil $(69,8 \%)$ y se conectan frecuentemente desde casa (94,9\%). Sin embargo, existe una brecha de acceso, ya que la distribución de conexión y dispositivos tecnológicos es muy desigual según el nivel socioeconómico de los alumnos (Fernández-Enguita, 2020), como se recoge en el Informe PISA 2018: el 14\% de los alumnos de nivel socioeconómico bajo no tiene ordenador en casa, y el $44 \%$ solo tiene uno. Las cifras contrastan con alumnos de nivel socioeconómico alto donde el $61 \%$ de los alumnos dispone de 3 o más ordenadores en casa. Se añade una brecha por falta de competencia (Van-Deursen \& Van-Dijk, 2010) que se suma a las variables demográficas (Kennedy et al., ( 2010) y a las disciplinas temáticas, diseño pedagógico de los cursos, situación socioeconómica de los estudiantes y sus circunstancias de vida (Margaryan et al., (2011). Los menores pueden usar la tecnología o las redes sociales, pero sin tener una competencia digital plena y sin estar acompañados en ellas son huérfanos digitales (Cabrera-Méndez \& DíezSomavilla, 2017).

\subsection{Papel e implicación de los docentes en la configuración del nuevo entorno educativo}

Las TIC dinamizan el proceso de enseñanza-aprendizaje (Díez-Somavilla, 2016; Colás-Bravo et al., ( 2018), sin embargo, su uso en contextos de formación es limitado y centrado en la motivación, la transmisión de 
información y en la comunicación (García-Valcárcel \& Alonzo-Mendoza, 2008; Fernández-de-la-Iglesia, 2012). Los propios docentes señalan el bajo grado de formación en TIC en su utilización didáctica y metodológica y manifiestan la necesidad formativa (García-Valcárcel \& Tejedor-Tejedor, 2010; Almerich-Cerveró et al., (2011; Gutiérrez-Porlán, 2014). Diversos estudios reflejan la necesidad de formación por parte del docente como una variable crítica (Sigalés et al., (2008; Mueller et al., ( 2008). Incluso señalan la escasa formación que reciben en la competencia digital los futuros docentes en las Facultades de Educación (Prendes-Espinosa et al.,( 2010).

A la carencia y competencias en TIC, se suma la falta de desarrollo de procedimientos didácticos en el desarrollo de la competencia digital del alumnado (Fernández \& Fernández, 2016). Aunque los docentes muestran interés en la formación tecnológica (Almerich et al., 2011), es el uso de metodologías y estrategias específicas lo que convierte los entornos en significativos para el aprendizaje y la innovación educativa (Guerrero-Pulido \& GisbertCervera, 2012). El IV Estudio sobre el uso de la tecnología en la educación en España (2018) destaca que uno de los mayores retos en la introducción de las TIC en las aulas es la formación del profesorado (44\%) y adaptar el proceso de aprendizaje-educativo al uso de la tecnología (31\%).

Existe una resistencia al cambio y a la innovación en la educación (Álvarez-Méndez, 2001). Las escuelas no disponen de un proyecto consensuado en la utilización de las TIC y muchas de las didácticas con tecnologías digitales, no suponen una mejora de las prácticas tradicionales (Christian \& Mathrani, 2014). Sepúlveda-Ruiz y Calderón-Almendros (2007) afirman que en la mayoría de los casos el único cambio apreciable es de tipo material, al sustituir parcialmente el uso de libros por ordenadores, pero manteniendo la metodología anterior. En el estudio de Losada-Iglesias et al., (2017) se concluye que los recursos didácticos tradicionales son los más utilizados.

\subsection{La implicación de las familias en la nueva enseñanza}

La implicación de las familias en la escuela y en la educación es un indicador de la calidad de la enseñanza, lo que lleva a impulsarla y favorecerla (Colás-Bravo \& Contreras-Rosado, 2013). Esta participación es uno de los pilares del sistema educativo, (Andrés-Cabello \& Giró-Miranda, 2016). Numerosos estudios han demostrado la correlación positiva entre la implicación de los padres en la educación de sus hijos y los resultados escolares y académicos (Egido, 2015; Bolívar, 2006; Ballesteros-Velázquez et al., (2014); participación que se ha hecho obligatoria en tiempos de confinamiento.

Algunos estudios (Aesaert \& Van Braak, 2014; Ames, 2016) indican que la vida digital en la familia, su nivel cultural y económico influye en la competencia digital de los menores. El nivel socioeconómico y cultural de los padres puede explicar la competencia digital del alumno (Sánchez-Antolín et al., (2018). También el nivel de estudios de los padres, la cantidad de ordenadores en el hogar y la calidad del acceso a Internet; a mayor nivel de estudios, se tiene más recursos y mayor conocimiento TIC (INE, 2019; Segura-Escobar, 2007).

\section{Metodología}

El estudio es una investigación descriptiva a través de una encuesta anónima de percepción mediante preguntas cerradas (datos primarios: https://bit.ly/370Yshq). La encuesta se ha utilizado para la búsqueda sistemática de información, en la que se cuestiona sobre los datos con el objetivo de juntar datos individuales y obtener datos agregados. Se trata de "obtener, de manera sistemática y ordenada, información sobre las variables que intervienen en la investigación, y esto sobre una población determinada» (Visauta, 1989: 259). La investigación se realizó desde el día 22 al 29 de abril de 2020, en pleno confinamiento nacional, decretado el 14 de marzo, con el objetivo de recopilar la experiencia tras el primer mes y medio en cuarentena y obtener una muestra de la opinión de los padres al inicio de la formación digital. 


\subsection{Muestra y muestreo}

La encuesta se compartió a través de medios digitales (WhatsApp, Facebook, Twitter, Linkedln y correo electrónico), y permitió, mediante un muestreo aleatorio simple, obtener una muestra (https://bit.ly/2TIEltg) de 2.150 individuos de toda España (81\% madres y 19\% padres). El número de escolarizados en educación obligatoria en España fue de 4.953.201 en 2019; teniendo en cuenta que la media de hijos en España es de 1.31 (INE, 2019); se ha tomado como tamaño de la muestra un total de 3.781.069, que corresponde a las familias que entrarían a formar parte del estudio. Para obtener un nivel de confianza del $95 \%$ y un margen de error del 5\%, se deberían haber realizado un mínimo de 385 encuestas, mínimo ampliamente superado.

La muestra tiene un alto nivel de estudios ( $46.4 \%$ universitarios, $12.4 \%$ postgrado y $4.4 \%$ doctorado), que difiere del nivel de estudios de la media nacional (Ministerio de Educación y Formación Profesional, 2019), por lo que no debemos tomar los datos como representativos del total de la población española (figura 1), sino de un nivel sociocultural alto. Este hecho se explica por el propio formato de la encuesta. En plena pandemia mundial, se utilizaron medios digitales para compartir la encuesta. La proporción de respuesta por nivel de estudios se puede explicar por la brecha de acceso y la brecha de uso según el nivel socioeconómico de las familias (Zubillaga \& Gortazar, 2020).

\section{Figura 1}

Nivel de estudio de la población española y de la muestra

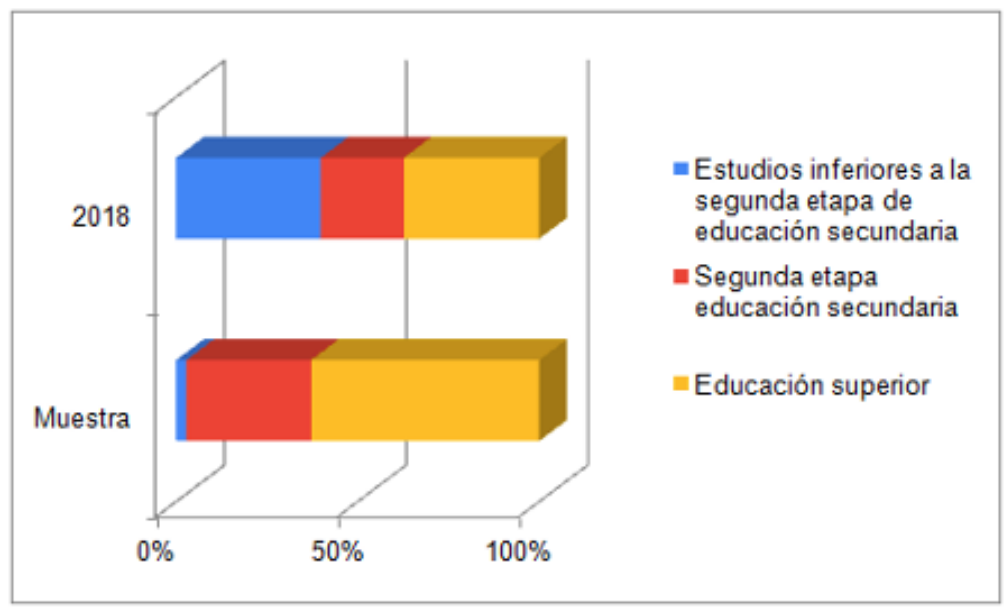

Fuente: Elaboración propia

El cálculo de la muestra estadística se ha realizado con la siguiente fórmula:

$$
\text { Tamaño de la muestra }=\frac{\frac{z^{2} \times p(1-p)}{e^{2}}}{1+\left(\frac{z^{2} \times p(1-p)}{e^{2} N}\right)}
$$

$\mathrm{N}=$ tamaño de la población $\bullet \mathrm{e}=$ margen de error $($ porcentaje expresado con decimales $) \bullet \mathrm{z}=$ puntuación $\mathrm{z}$

La puntuación z es la cantidad de desviaciones estándar que una proporción determinada se aleja de la media, en este caso se ha considerado que para obtener el 95\% la puntuación z es de 1,96. 


\subsection{Procesamiento y análisis de datos}

Se desglosan los datos agregados en bloques por tipo de centro (https://bit.ly/2TO4Lzp) al que asisten cada uno de los hijos de los encuestados (58.9\% centros públicos, $33 \%$ concertados y $8 \%$ privados) y nivel de estudios (62.3\% en primaria y $37.6 \%$ en secundaria), para tener un enfoque objetivo e imparcial, una base relacional y trabajar los datos por grupos de interés, y así obtener grupos de variables. A raíz de los datos que mejor definen la apreciación de las familias se agrupan por bloques: grado de satisfacción de las familias; y apreciación sobre la docencia y metodología del sistema de enseñanza online.

Para el análisis estadístico se usó el software Prism 5 de GraphPad software. Un valor estadístico de P menor a .05 se consideró significativo estadísticamente; y se compararon los valores usando una tabla de contingencia y el test de Chi-cuadrado.

\section{Resultados}

\subsection{Grado de satisfacción de las familias}

El grado de satisfacción de las familias, con respecto a las metodologías y a la docencia que están recibiendo los hijos figura 2) en primaria, destacan los centros privados; las familias están satisfechas en un $60 \%$ de los casos, mientras que las familias de centros concertados lo están en un $44 \%$, y las que los llevan a centros públicos en un $37 \%$. Esta diferencia es estadísticamente significativa ( $p=.00225)$. En secundaria ocurre lo mismo, destacando todavía más los centros privados, con un nivel de satisfacción del 71\%, mientras que en la pública solo es del $45 \%$ $(p<.0001)$.

Se preguntó por el grado de satisfacción de las familias con los docentes, teniendo en cuenta la situación. Las respuestas para primaria y secundaria son muy similares; destacamos, que los docentes de centros privados son mejor valorados, ya que el $80 \%$ de las familias están muy satisfechas, comparado con un $60 \%$ de satisfechos con la concertada, o un $55 \%$ de la pública. Mientras que las familias que tienen un nivel bajo de satisfacción con los docentes, o les «suspenden» solo son el $10 \%$ de los que llevan a sus hijos a centros privados, un $15 \%$ de los concertados y $20 \%$ en públicos.

Gráfico 2

Grado de satisfacción de los padres con las metodologías y la docencia que actualmente está recibiendo su hijo

(donde 1 es muy decepcionado y 5 muy satisfecho)

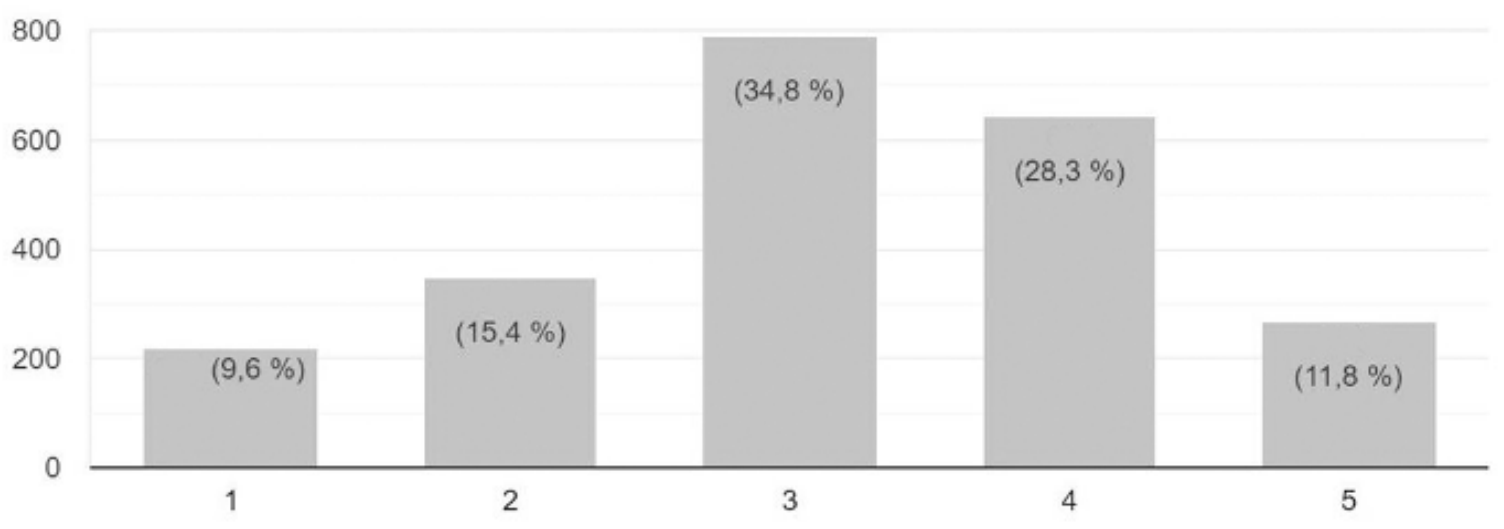

Fuente: Elaboración propia.

Sobre la apreciación de cómo sus hijos están viviendo la nueva situación de docencia online, cerca del 38\% perciben que sus hijos están igual que antes, independientemente del tipo de centro o el nivel de enseñanza. 
Entre los menores a los que sus padres notan más aburridos que antes, el $41 \%$ de familias en centros públicos les siente aburridos; un $36 \%$ en los concertados; y solo un $24 \%$ en los privados. Al observar si notan a sus hijos más felices que antes, en los centros privados los padres señalan que en primaria están un $35 \%$ más felices y los de secundaria de centros privados un $43 \%$. Los niños de centros concertados de primaria y secundaria están más felices en un $30 \%$; y los de centros públicos de primaria en un $22 \%$, y un $24 \%$ en secundaria.

Sobre si existe la interrelación de los menores en el aula virtual, el 85\% de los padres con hijos en primaria en centros privados afirman que sí se están relacionando con los compañeros; en centros concertados la cifra desciende al 51\%; y al $35 \%$ en los centros públicos $(p<.0001)$. En secundaria los resultados son similares, destacando los centros privados, con un $89 \%$, mientras que en la pública solo es del $51 \%(p<.0001)$.

A la pregunta de cuánto considera que están trabajando los menores durante el confinamiento (clases + tareas), si es más o menos que antes, la mayoría con hijos en primaria y en secundaria, independientemente del tipo de centro, consideran que sus hijos trabajan menos durante la cuarentena. En cuanto a las horas al día dedicadas al aprendizaje, destacan notablemente los centros privados mostrando más carga laboral, tanto en primaria como en secundaria: dedicando la mayoría entre 4 y 6 horas. Mientras que los centros de educación primaria y secundaria, públicos y concertados, muestran porcentajes similares, predominando el intervalo de 2-4 horas de trabajo diario. Esta diferencia es significativa en los dos niveles $(p<.0096)$.

\subsection{Apreciación sobre la docencia y metodología de la enseñanza online}

En relación al acceso a un equipo propio para realizar las tareas escolares, los datos reflejan una diferencia de casi 30 puntos porcentuales entre los centros públicos y privados a favor de los últimos, diferencia menos marcada en los centros concertados. La práctica totalidad de estudiantes de primaria y secundaria de centros privados disponen de un equipo propio para hacer las tareas. A grandes rasgos la diferencia porcentual entre tipos de centro se mantiene de primaria a secundaria, aunque aumenta la tasa de estudiantes, independientemente del tipo de centro, con equipo propio. Los centros públicos aglutinan las menores tasas de disponer de equipo propio.

El profesorado de primaria trabaja siguiendo el horario del curso; envía o sube material de trabajo coincidiendo con los días de clase presencial, sobre todo en los centros privados (74\%) y en los concertados (61\%). En cambio, en los centros públicos de primaria este porcentaje es del $51 \%$. El comportamiento en la ESO es similar, pero con peores resultados para los centros públicos, solo el $35 \%$ de los profesores suben material coincidiendo con el calendario presencial, frente al $76 \%$ de los privados y el $64 \%$ de los concertados.

La docencia a distancia se realiza con el material educativo habitual (libros y ejercicios), pero de forma complementaria en primaria los docentes crean material específico. Esta tendencia se observa también en la ESO. Debido a la edad de los estudiantes (12-16 años) existe un porcentaje destacable de padres que desconocen si los docentes crean material específico para sus hijos, puesto que son más independientes que en primaria.

Sí hay unanimidad entre las familias en conocer con antelación las fechas de entrega de las tareas para poder planificarse. En los centros de primaria observamos la figura del coordinador de tareas, que sigue una metodología acorde a los objetivos del curso. El porcentaje de dicha coordinación es alto y similar entre diferentes centros (en torno al 70\%). En cambio, en secundaria las encuestas desprenden una menor coordinación en centros públicos. Datos más positivos ofrece la enseñanza concertada y privada. Tanto en primaria como en secundaria encontramos tasas relevantes de desconocimiento de fechas de entrega por parte de los padres (en torno al 16\%).

La coordinación de las diferentes materias está siendo difícil, principalmente en la ESO donde el número de asignaturas es mayor y cada una de ellas depende de un profesor. Los padres de primaria valoran positivamente 
la coordinación y organización entre materias y profesores, aunque algo peor valorada en los colegios públicos. Sin embargo, la percepción de las familias de esta coordinación cae en secundaria. Al igual que en el resto de las acciones educativas, existe un importante desconocimiento por parte de las familias (en torno al 18\% independientemente del centro). La percepción familiar sobre coordinación en los centros concertados es más positiva (53\%), se incrementa en los privados $(66 \%)$ y cae en los públicos $(28 \%)$.

Los padres de alumnos en primaria de centros concertados y públicos consideran que sus hijos desconocen en gran medida qué deben hacer sin ayuda de un adulto en casa (62\%), valor más equitativo en centros privados (50\%), aunque la diferencia no es significativa. Estas cifras sí son significativas en secundaria $(p=.0047)$, donde el $70 \%$ de alumnos en centros privados saben qué hacer sin explicación previa en el hogar, un $61 \%$ en concertados y un $45 \%$ en públicos.

Sobre el sistema de evaluación empleado durante la COVID-19, en primaria, en los tres tipos de centros, hay un gran desconocimiento de los padres sobre la forma de evaluar a sus hijos, entre el $64 \%$ y el $77 \%$ desconocía cómo iban a ser evaluados. En secundaria, sí hubo diferencia significativa $(p<.0001)$ : más de la mitad de los padres con niños en colegios privados conocían el sistema de evaluación, frente al $25 \%$ en concertados o el $29 \%$ en públicos.

En cuanto a si los centros tenían sistemas de docencia online antes de la crisis, destacan los centros concertados, tanto en primaria como en secundaria. Estos centros tenían más plataformas virtuales y las utilizaban en mayor proporción antes de la pandemia. En secundaria observamos diferencias más marcadas entre tipos de centros, aunque siguen destacando los centros concertados. Al igual que en la enseñanza primaria, un número relevante de progenitores desconocía si el centro de su hijo contaba con una plataforma virtual antes de la crisis.

En cuanto a si en la crisis los centros disponen de una plataforma virtual, destacan los centros privados, puesto que tanto en primaria como en secundaria más del $92 \%$ disponen de una. En los centros de secundaria la gran mayoría (>80\%) cuentan con uno, mientras que en primaria los centros públicos solo un $48 \%$ tienen. Los datos muestran una reducción general del desconocimiento por parte de los progenitores sobre la existencia de una plataforma virtual.

\section{Conclusiones}

El grado de satisfacción de las familias con la reciente implantada formación online en los menores que cursan educación obligatoria en España difiere dependiendo del nivel y del centro al que acuden. Los resultados indican que el nivel de satisfacción es mucho mayor en familias que llevan a sus hijos a centros privados respecto a centros concertados o públicos; y dentro de esta diferenciación, también están mejor valorados la coordinación de los docentes y el sistema de enseñanza en el nivel de primaria, que en el de secundaria. Sin embargo, la gran mayoría de padres, independientemente del tipo de centro, valora la implicación y esfuerzo que están haciendo los docentes en la crisis del COVID-19, sobre todo en primaria.

La brecha digital es una realidad que afecta en mayor medida a los centros públicos, frente a los concertados y privados. Se crea así un eje público-privado donde, salvo alguna excepción puntual, los centros privados salen mejor parados en acceso a la tecnología, interrelación con los compañeros y disposición de sistemas digitales en sus centros; también en la organización y coordinación de las materias por parte de los docentes. En este eje, los centros concertados suelen encontrarse a medio camino entre sus homólogos, aunque la tendencia los ubica más cercanos a los centros privados.

En cuanto a las diferencias entre primaria y secundaria, los centros muestran las mismas diferencias porcentuales entre los tres tipos de centros. La peor parada es la ESO pública, donde encontramos menor coordinación entre asignaturas, materias y docentes; es decir, falta una coordinación general. Aspecto clave, sobre todo teniendo en cuenta la no presencialidad, es la figura de un coordinador de las tareas siguiendo una metodología y acorde 
a los objetivos del curso: el tutor; el encargado de facilitar los grupos de aprendizaje, aconsejar, planificar, motivar y supervisar el progreso del alumnado. Puede ser la razón por la que los padres de los estudiantes de secundaria de centros públicos son los que menos satisfechos están con la metodología empleada.

Sobre la disposición de una plataforma virtual los peores parados son los alumnos de primaria de centros públicos. Los padres consideran que sus hijos están más contentos y se aburren menos, coincidiendo con el nivel de uso de las plataformas de formación en línea; también se observa que consideran que trabajan mucho menos que antes, en general todos los alumnos, pero sobre todo los que no utilizan plataformas y acuden a centros públicos. Se recomienda que los centros utilicen aulas virtuales para que los alumnos tengan un espacio virtual donde cohesionar la enseñanza en línea, puedan planificar mejor el tiempo e interelacionarse con compañeros y docentes.

Existe un notable grado de desconocimiento por parte de las familias, tanto sobre las metodologías como sobre la existencia de plataformas digitales. Este desconocimiento sobre el desarrollo de la educación digital en los hijos es más marcado en los estudios de ESO; desentendiéndose de las tareas o del tiempo que están empleando sus hijos en la educación en línea. La mayoría de las familias también desconoce la forma en la que se va a evaluar a sus hijos, aunque hay que tener en cuenta que la encuesta se hizo previa a que el Ministerio de Educación y Formación Profesional publicara (BOE, 24-4-2020) unas directrices sobre la evaluación.

Habrá que seguir la evolución de esta satisfacción y analizarla en el tiempo, ya que es probable la vuelta a algún tipo de confinamiento o la combinación de una enseñanza presencial y en línea. Los centros deberían estar preparados con guías docentes adaptadas, los docentes tendrán que coordinarse mejor y los alumnos adquirir competencias digitales que les hagan ser más efectivos. También habrá que equiparar la enseñanza síncrona y asíncrona, y el número de horas de enseñanza efectiva para que no existan tantas desigualdades entre unos centros y otros.

Resaltamos como una limitación de este estudio que en la muestra participaron familias con un nivel de estudios alto y con conocimientos en el uso de las TICs. Por esta razón, se requieren otros estudios que analicen qué está ocurriendo con familias más desfavorecidas ya que, el impacto de la formación online precipitada seguramente haya sido diferente en estos dos contextos. No obstante, este artículo es uno de los primeros desarrollos empíricos en evaluar la apreciación de las familias en este nuevo entorno educativo.

\section{Referencias bibliográficas}

Aesaert, K., y Van Braak, J. (2014). Exploring factors related to primary school pupils' ICT self-efficacy: A multilevel approach. Computers in Human Behavior, 41, 327-341. doi: 10.1016/j.chb.2014.10.006

Ala-Mutka, K. (2011). Mapping Digital Competence: Towards a Conceptual Understanding. IPTS, European Commission. Luxembourg: European Commission. Recuperado de https://bit.ly/2zw7u9Q

Almerich-Cerveró, G., Suárez-Rodriguez, J., Jornet-Meliá, J., y Orellana-Alonso, N. (2011). Las competencias y el uso de las Tecnologías de la Información y Comunicación por el profesorado: estructura dimensional. Revista Electrónica de Investigación Educativa, 13(1), 28-42. Recuperado de https://bit.ly/3da7B90

Álvarez-Méndez, J.M. (2001). Entender la didáctica, entender el currículum. Miño y Dávila. Madrid.

Ames, P. (2016). Los niños y sus relaciones con las tecnologías de información y comunicación: un estudio en escuelas peruanas. DESIDADES - Revista Eletrônica de Divulgação Científica Da Infância E Juventude, 11, 11-21. Recuperado de https://bit.ly/2zxMdws 
Andrés-Cabello, S., y Giró-Miranda, J. (2016). La participación de las familias en la escuela: una cuestión compleja. Revista de Evaluación de Programas y Políticas Públicas 7, 28-47. doi:10.5944/reppp.7.2016.16302.

Ballesta-Pagan, F., Izquierdo-Rus, T., y Romero-Sánchez, B. (2011). Percepción del alumnado de pedagogía ante el uso de las metodologías activas. Educatio Siglo XXI, 29 (2), 353-368. Recuperado de https://bit.ly/2znVORL

Ballesteros-Velázquez, B, Aduado-Odina, y T. Malik-Liévano, B. (2014). Escuelas para todos: diversidad y educación obligatoria. Revista Electrónica Interuniversitaria de Formación del Profesorado, 17(2), 93-107. doi: 10.6018/reifop.17.2.197351

Blanco, I., y Römer, M. (2011). Los niños frente a las pantallas. Madrid: Universitas.

Blink Learning (Ed.) (2018). Estudio sobre el uso de la tecnología en la educación (IV edición). Recuperado de https://bit.ly/3gp8ehx

Bolívar, A. (2006). Familia y escuela: dos mundos a trabajar en común. Revista de Educación, 339, 119-146. Recuperado de https://bit.ly/36zkfwc

Bringué-Sala, X., y Sábada-Chalezquer, C. (2010). Niños y adolescentes españoles ante las pantallas: rasgos con curadores de una generación interactiva. CEE Participación Educativa, 86-104. Recuperado de https://bit.ly/2B3kJPL

Cabero-Almenara, J., y Marín-Díaz, V. (2014). Miradas sobre la formación del profesorado en TIC. Enl@ce, 11(2), 11-24. Recuperado de https://bit.ly/2X4veuQ

Cabra-Torres, F., y Marciales-Vivas, G.P. (2009). Mitos, realidades y preguntas de investigación sobre los 'nativos digitales': una revisión. Universitas Psychologica, 8(2), 323- 338. Recuperado de https://bit.ly/2B3kJPL

Cabrera-Méndez, M., y Díez-Somavilla, R. (2017). Padres analógicos frente a huérfanos digitales. En S. Lluna, y Pedreira (Coords.), Los nativos digitales no existen (pp. 117 - 135). Deusto.

Cabrera-Méndez, M., Poza-Luján, J.L., y Lloret-Romero, N. (2019). Docentes: mutación o extinción. Revista Telos, Fundación Telefónica. Recuperado de https://bit.ly/36uKRhW

Christian, S., y Mathrani, A. (2014). ICT Education: Socio-Learning Issues Faced by International Students. Proceedings of the Thirty Fifth International Conference on Information Systems, Auckland, New Zealand. Recuperado de https://bit.ly/3efZEzG

Cobo, C., y Moravec, J.W. (2011). Aprendizaje invisible. Hacia una nueva ecología de la educación. Barcelona: Laboratori de Mitjans Interactius / Publicacions i Edicions de la Universitat de Barcelona, Col.lecció Transmedia XXI. Recuperado de https://bit.ly/3gvAHT9

Colás-Bravo, P., y Contreras-Rosado, J.A. (2013). La participación de las familias en los centros de Educación Primaria. Revista de Educación Educativa, 31(2), 485-499. doi: 10.6018/rie.31.2.171031

Colás-Bravo, M.P., De-Pablos-Pons, J., y Ballesta-Pagán, J. (2018). Incidencia de las TIC en la enseñanza en el sistema educativo español: una revisión de la investigación. Revista de Educación a Distancia, 56, (2). doi: $10.6018 / \mathrm{red} / 56 / 2$

Coombs, P.H. (1978). La crisis mundial de la educación. Península. 
Cooper, H., Nye, B., Charlton, K., Lindsay, J., y Greathouse, S. (1996). The effects of summer vacation on achievement test scores: A narrative and meta-analytic review. Review of Educational Research, 66(3), 227-268. doi: 10.3102/00346543066003227

Darling-Hammond, L. (2000). Teacher Quality and Student Achievement: A Review of State Policy Evidence. Educational Policy Analysis Archives, 8(1). Recuperado de https://bit.ly/2ZA2J9X

Díez-Somavilla, R. (2016). Los valores educativos y las competencias en los videojuegos de la TDT infantil española [Tesis doctoral]. Universitat Politècnica de València. doi: 10.4995/Thesis/10251/68484

Egido, I. (2015). Las relaciones entre familia y escuela. Una visión general. Participación Educativa, 7, 11-17. doi: 10.4438/1886-5097-PE

Feixa, C. (2006). Generación XX. Teorías sobre la juventud en la era contemporánea. Revista Latinoamericana de Ciencias Sociales, Niñez y Juventud, 4(2), 21-45. Recuperado de https://bit.ly/2zzjUxz

Fernández-Cruz, F.J., y Fernández-Díaz, M.J. (2016). Los docentes de la Generación Z y sus competencias digitales. [Generation Z's Teachers and their Digital Skills]. Comunicar, 46, 97-105. doi: 10.3916/C46-201610

Fernández-de-la-Iglesia, J. C. (2012). Competencias TIC de los docentes para la sociedad del Conocimiento [Tesis doctoral inédita]. Santiago de Compostela, Facultad de Educación.

Fernández-Enguita, M. (2020, marzo 31). Una pandemia imprevisible ha traído la brecha previsible. Cuaderno de Campo. Recuperado de https://bit.ly/2X2SnOr

García-Valcárcel, A., y Alonzo-Mendoza, M. D. (2008). La integración de las tecnologías de la información y comunicación en la enseñanza universitaria: cómo afrontan los profesores el cambio al espacio europeo de educación superior. En R. Roig. (dir.). Investigación e innovación en el conocimiento educativo actual. (pp. 129-166). Marfil: Alcoy

García-Valcárcel, A., y Tejedor-Tejedor, F. J. (2010). Evaluación de procesos de innovación escolar basados en el uso de las TIC desarrollados en la Comunidad de Castilla y León. Revista de Educación, 352, 125- 147. Recuperado de https://bit.ly/36vfPXm

González-Peiteado, M., y Pino-Juste, M. (2016). Los estilos de enseñanza: construyendo puentes para transitar las diferencias individuales del alumnado. Revista Complutense de Educación, 27(3), 1175-1191. doi: 10.5209/rev_RCED.2016.v27.n3.47563

Gilster, P. (1997). Digital literacy. New York: Wiley.

Guerrero-Pulido, J.F., y Gisbert-Cervera, M. (2012). El cambio organizacional en la Universidad a través del uso de los campus virtuales desde la perspectiva de los estudiantes. Píxel-Bit. Revista de Medios y Educación, 40, 75-88. Recuperado de https://bit.ly/2THH1gj

Gutiérrez-Porlán, I. (2014). Perfil del profesor universitario español en torno a las competencias en tecnologías de la información y la comunicación. Píxel-Bit. Revista de Medios y Educación, 44, 51-65. doi:

10.12795/pixelbit.2014.i44.04

Hodges, Ch., Moore, S., Lockee, B., Trust, T., y Bond, A. (2020). The Difference Between Emergency Remote Teaching and Online Learning. EducaseReview. Recuperado de https://bit.ly/3goAild 
Informe PISA (Ed.) (2018). Programa para la Evaluación Internacional de los Estudiantes. Ministerio de Educación y Formación Profesional. Recuperado de https://bit.ly/36wIQBQ

Instituto Nacional de Estadística (Ed.) (2019). Encuesta sobre equipamiento y uso TIC en los hogares. Secretaria de Estado de Economía y Apoyo a la Empresa. Recuperado de https://bit.ly/3gt2PpO

Jaume, D., \& Willen, A. (2019). The Long-Run Effects of Teacher Strikes: Evidence from Argentina. Journal of Labor Economics 37(4), 1097-1139. Recuperado de https://bit.ly/3gpSgny

Johnson, L., Adams-Becker, S., Estrada, V., Freeman, A., Kam py lis, P., Vuorikari, R., y Punie, Y. (2014). Horizon Report Europe: 2014 Schools Edition. Luxembourg: Publications Office of the European Union, \& Austin, Texas: The New Media Consortium. Recuperado de https://bit.ly/2LYxL30

Kennedy, G., Judd, T., Dalgarnot, B., y Waycott, J. (2010). Beyond natives and immigrants: Exploring types of net generation students. Journal of Computer-Assisted Learning, 26(5), 333-343. doi: 10.1111/j.13652729.2010.00371.x

Losada-Iglesias, D., Correa-Gorospe, J. M., y Fernández-Olaskoaga, L. (2017). El impacto del modelo «un ordenador por niño» en la Educación Primaria: Un estudio de caso. Educación XXI, 20(1), 339-361. doi: 10.5944/educXX1.17515

Margaryan, A., Littlejohn, A., y Vojt, G. (2011). Are digital natives a myth or reality? University students' use of digital technologies. Computers \& Education, 56(2), 429- 440._doi: 10.1016/j.compedu.2010.09.00

Mcloughlin, M. (2020, marzo 19). Casi toda España conectada desde casa: ¿puede aguantar Internet todo ese tráfico? El Confidencial. Recuperado de https://bit.ly/3d8byvp

Ministerio de Educación y Formación Profesional (Ed.) (2019). Sistema estatal de indicadores de la educación 2019. Recuperado de https://bit.ly/36Au3q7

Moore, M. G., y Kearsley, G. (2011). Distance Education: A Systems View of Online Learning (What's New in Education). Cengage Learning Custom Publishing.

Mueller, J., Wood, E., Willoghby, T, Ross, C., y Specht, J. (2008). Identifying discriminating variables between teachers who fully integrate computers and teachers with limited integration. Computers \& Education, 51(4), 1523-1537. doi: 10.1016/j.compedu.2008.02.003

Observatorio Nacional de las Telecomunicaciones y de la SI (Ed.) (2019). Indicadores sobre uso de TIC por menores en España (febrero). Recuperado de https://bit.ly/3c1eiJH

Pérez-Escoda, A., Castro-Zubizarreta, A. y Fandos-Igado, M. (2016). La competencia digital de la Generación Z: claves para su introducción curricular en la Educación Primaria. [Digital skills in the Z generation: Key questions for a curricular introduction in Primary School]. Comunicar 49, 71-80. doi: 10.3916/C49-201607

Prendes-Espinosa, M.P., Castañeda-Quintero, L., y Gutiérrez-Porlan, I. (2010). Competencias para el uso de TIC de los futuros maestros. [ICT competences of future teachers]. Comunicar, 35, 175-182. doi: 10.3916/C352010-03-11

Prensky, M. (2001). Digital Natives, Digital Immigrants. On the Horizon, 9(5), 1-6. Recuperado de https://bit.ly/2Xyz6TE 
Real Decreto 463/2020, por el que se declara el estado de alarma para la gestión de la situación de crisis sanitaria ocasionada por el COVID-19, 14 de marzo de 2020. BOE. Recuperado de https://bit.ly/2A88ilb

Sánchez-Antolín, P., Andrés-Viloria, C., y Paredes-Labra, J. (2018). El papel de la familia en el desarrollo de la competencia digital. Análisis de cuatro casos. Digital Education Review, 34. Recuperado de https://bit.ly/2AaBQP4

Sangrá, A., Vlachopoulos, D., y Cabrera, N. (2012). Building an inclusive definition of e-learning: An approach to the conceptual framework. The International Review of Research in Open and Distance Learning, 13(2), 145-159. doi: 10.19173/irrodl.v13i2.1161

Sanz, I. (2020, marzo 19). Efectos de la crisis del coronavirus sobre la educación. Magisterio. Recuperado de https://bit.ly/3d5yDiq

Segura-Escobar, M. (2007). Las TIC en la Educación: panorama internacional y situación española. Santillana.

Sepúlveda-Ruiz, M., y Calderón-Almendros, I. (2007). Las TIC y los procesos de enseñanza-aprendizaje: la supremacía de las programaciones, los modelos de enseñanza y las calificaciones ante las demandas de la sociedad del conocimiento. Revista Iberoamericana de Educación, 44(5), 1-13. doi: 10.35362/rie4452217

Sigalés, C., Mominó, C., Meneses, J., y Badía, A. (2008). La integración de internet en la educación escolar española: situación actual y perspectivas de futuro. Barcelona: UOC.

Tapscott, D. (1999). Educating the net generation. Educational Leadership, 56(5), 6-11.

Van-Deursen, A., y Van-Dijk, J. (2010) Internet Skills and the digital divide. New media \& society, 13(6) 893-911. doi: $10.1177 / 1461444810386774$

Visauta, B. (1989). Técnicas de investigación social I: recogida de datos. Promociones y publicaciones universitarias S. A., Barcelona

Zafra, I. (2020, mayo 5). El Gobierno planea limitar a 15 el número de alumnos por aula para el curso que viene. El País. Recuperado de https://bit.ly/3d80ZbU

Zubillaga, A., y Gortazar, L. (2020) (Coord.). Covid-19 y educación: problemas, respuestas y escenarios. Fundación COTEC para la innovación. Recuperado de https://bit.ly/3d78tf9

Esta obra está bajo una Licencia Creative Commons Attribución-NoCommercial 4.0 International

(cc) BY-NC 